\title{
Analyzing Ballistic Missile Defense System Effectiveness Based on Func- tional Dependency Network Analysis
}

\author{
Yao Jian", Huang Qiwang and Wang Weiping \\ College of Information System and Management, National University of Defense Technology, Hunan, Changsha, \\ 410073, P.R. China
}

\begin{abstract}
Recent engineering experiences highlight the Ballistic Missile Defense System (BMDS) as a System of Systems (SoS), and traditional probability models were found to be limited in capturing the complex interactions between component systems. A novel approach was proposed to evaluate the effectiveness of the whole BMDS from the aspect of functional interactions, rather than the functions of components. First, Functional Dependency Network Analysis (FDNA) was introduced to build the analysis model through integrating the component systems in multi-layered kill chains of BMDS. The key metrics were proposed to measure the effectiveness from both probability of raid annihilation and system response time. Finally, the application of this method was demonstrated through a case study, and the result showed the holistic characters of BMDS at SoS level. The case showed that FDNA was a promising way to analyze SoS at high level.
\end{abstract}

Keywords: Ballistic missile defense system, Effectiveness analysis, Functional dependency network analysis, System of systems.

\section{INTRODUCTION}

Ballistic Missile Defense System (BMDS) being developed, tested and deployed by the United States provides initial capability to counter ballistic missiles in multi-layers, while other countries (e.g., Russian and China) have also achieved great progress in missile defense capability.

Traditionally, the effectiveness analysis of BMDS was based on the simplified probability models of both intercept missiles and support sensors [1-3]. With continued attention to the architecture integrating sensors with shooters, specifically to implement launch-on-remote (LOR) and engage-onremote (EOR) firing doctrines [4], recent engineering researches focused on numerous interactions between the component systems of the BMDS in view of the system of systems $(\mathrm{SoS})[5,6]$.

The most critical interactions in SoS are functional dependencies between the component systems, as the mission of SoS is completed by the whole not by a single or several component systems, and the functional dependencies integrate the components as a whole. Therefore, it is necessary to evaluate the effectiveness of BMDS from the aspect of functional dependencies.

To assess the effectiveness of BMDS at the level of SoS, the study built the functional dependency network (FDN) of BMDS through integrating the kill chains in multi-layered defense system to form the metrics to assess and measure the effectiveness of BMDS.

\footnotetext{
*Address correspondence to this author at the College of Information System and Management, National University of Defense Technology, Hunan, Changsha, 410073, P.R. China; Tel: +86 073184573558 ; Fax: +86073184573558; E-mail: markovyao@163.com
}

The paper is organized as follows: In section 2, the current application of the effectiveness analysis of BMDS is reviewed. In Sections 3, functional dependency analysis model of BMDS is proposed. In section 4, a case study is presented to show how the outputs of the method can be used to quantify the metrics of effectiveness in BMDS. In section 5, conclusions are drawn with some recommendations for applications and future work.

\section{RELATED WORK}

\subsection{BMDS Overview}

The BMDS consists of a network of satellites, radars, Aegis ships, missile launchers and missiles, and provides intercepting capability in three phases of flight: boost phase, midcourse phase, and terminal phase, that form a multilayered missile defense system [4]. Whereas the currently deployed BMDS ensures missile defense with elements acting independently, and the next generation BMDS focuses on the capability of distributed engagements: EOR and LOR [4]. In the LOR concept, the missile launching ship receives input from off-board sensors (for example, land-based AN/TPY-2 radar) of good quality to launch an interceptor and do not need to acquire the target track on its on-board sensors at the time of launching. EOR is an extension of LOR, in which the interceptor can be launched using any available target track and engagement is controlled from inflight target updates that can be provided to the interceptor missile from any Aegis AN/SPY-1 or AN/TYP-2 radar [5].

\subsection{BMDS Effectiveness Analysis}

Probabilistic model is widely applied in the analysis of the effectiveness of BMDS from the aspects of configuration and doctrine [3]. Menq introduced a discrete time Markov 
process model to multi-layered BMD system [1]. Wilkening proposed a simple Bernoulli trials model to determine the size of required BMDS [2]. The effectiveness of BMDS was presented by the probability distribution of the number of penetrating warheads, and optimal fire doctrines were proposed based on these models. Probabilistic model focused on the capability of interceptor, however, the support systems (e.g. radar sensors, communications networks) were ignored or simplified, and failed to capture the internal dependency between the component systems.

Due to the complexity of BMDS, the system of systems engineering is applied to study the effectiveness [6]. Tommer built surrogate models of components in BMDS, and provided an M\&S environment for real-time, high-level BMDS architecture trade studies at the SoS level [7]. Garrett proposed an SoS framework to manage the interstitials, in which Graph Theory was applied to analyse information flowing between the component systems [5]. Agent Based Modeling (ABM) was proposed as a technique to explore and quantify the interstitial behaviors [8]. Most system of systems engineering adopted M\&S approach to model the complex interactions and focused on high-fidelity component models. However, interaction as a key factor to form SoS capability was not studied independently .

\section{FUNCTIONAL DEPENDENCY NETWORK OF BMDS}

\subsection{Basic of FDNA}

Functional Dependency Network Analysis (FDNA) was originally formulated by Garvey [9], who applied it to carry out capability portfolio analysis and risk assessment. FDNA has been applied in the SoS analysis for wide domains. Guariniello extended FDNA in both operational and development networks to analyse the complex dependencies between the systems and capabilities of SoS, applied in the aerospace SoS design and architecture [10]. Drabble [11] applied the dependency network analysis to identify the key dependent nodes and conduits within the information propagation network for emergency response. Wang [12] and Zhang [13] analyzed the SoS security of Global Navigation Satellite Systems (GNSS) based on FDNA technique.

Accordingly $[9,10]$, in the FDN, the nodes represent systems or capabilities, while the links represent the operational dependencies between the systems or between the capabilities. Each dependency is characterized by strength (Strength of Dependency, SoD) and criticality (Criticality of Dependency, $\mathrm{CoD}$ ), that affect the behavior of the whole SoS in different ways. As in the SoS, CoD quantifies the operational independence of the components, while SoD represents the effect of interactions between the components. Those inputs can be achieved from expert judgment and evaluation, or the result of statistics of simulations and experiments. The formation of FDN is given as follows:

Define 1. A Functional Dependency Network (FDN) is a tuple $<\mathrm{V}, \mathrm{E}, \mathrm{C}, \mathrm{OE}>$, with $\mathrm{G}=(\mathrm{V}, \mathrm{E})$ representing a directed-acyclic graph, where

$\mathrm{V}=$ set of nodes representing either the component systems or the capability to be acquired,
$\mathrm{E}=$ set of edges representing the operational dependencies between nodes,

$\mathrm{C}=$ represents the dependencies attributes : $\mathrm{E} \rightarrow\{\mathrm{SoD}, \mathrm{CoD}\}, \mathrm{SoD} \in[0,1]$ is the strength of dependency fraction, and $\mathrm{CoD} \in[0,100]$ is the criticality of dependency constraint.

$\mathrm{OE}=$ represents the effectiveness level of the operation: $\mathrm{CoD} \rightarrow[0,100]$

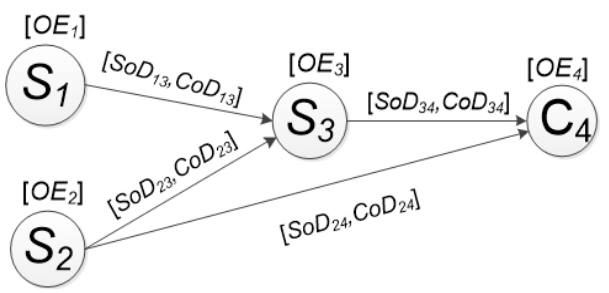

Fig. (1). An example of functional dependency network.

The paper presented an Breadth-First-Search algorithm to solve the FDNA, and the detailed calculation of the FDNA, which can also be found in [9], is shown the algorithm.

Algorithm 1. FDNA algorithm.

\begin{tabular}{|c|c|}
\hline \multicolumn{2}{|c|}{ Procedure FDNA $<V, E, C, O E>$} \\
\hline Function Anal & $\operatorname{alysis}(V)$ \\
\hline \multicolumn{2}{|c|}{ while $V \neq \varnothing$ do } \\
\hline \multicolumn{2}{|c|}{ for all $v_{i} \in V$ do } \\
\hline \multicolumn{2}{|c|}{ if $\mathrm{P}\left(v_{i}\right)=\varnothing$ then } \\
\hline \multicolumn{2}{|c|}{$V=V-v_{i}$} \\
\hline \multicolumn{2}{|c|}{$/ / \mathrm{P}\left(v_{i}\right)$ is the parent nodes set of $v_{i}$} \\
\hline \multicolumn{2}{|c|}{ else if $\mathrm{P}\left(v_{i}\right) \cap V=\varnothing$ then } \\
\hline \multicolumn{2}{|r|}{ Analysis $\left(\mathrm{P}\left(v_{i}\right) \cap V\right)$} \\
\hline \multicolumn{2}{|l|}{ else } \\
\hline \multirow{4}{*}{\multicolumn{2}{|c|}{$\begin{array}{l}\left.S o D_{-} P_{i}=\frac{1}{K_{v_{k} \in P\left(v_{i}\right)}} \sum_{k o D_{k i}} * O E_{k}+\left(1-S o D_{k i}\right) * O E_{i}\right) \\
C o D_{-} P_{i}=\underset{v_{k} \in P\left(v_{i}\right)}{\operatorname{Min}\left(C o D_{k i}+O E_{i}\right)} \\
O E_{i}=\operatorname{Min}\left(\operatorname{SoD} P_{i}, C o D_{-} P_{i}\right) \\
/ / K \text { is the number of nodes in } \mathrm{P}\left(v_{i}\right)\end{array}$}} \\
\hline & \\
\hline & \\
\hline & \\
\hline \multicolumn{2}{|l|}{ end if } \\
\hline \multicolumn{2}{|l|}{ end for } \\
\hline \multicolumn{2}{|l|}{ end while } \\
\hline \multicolumn{2}{|l|}{ end Function } \\
\hline end Procedure & \\
\hline
\end{tabular}

\subsection{Integrate BMDS Kill Chains into FDN}

As this study focused on the effectiveness of the whole system of systems, the FDN of BMDS was built by integrating multi-layered kill chains. In the proposed FDNA model, 


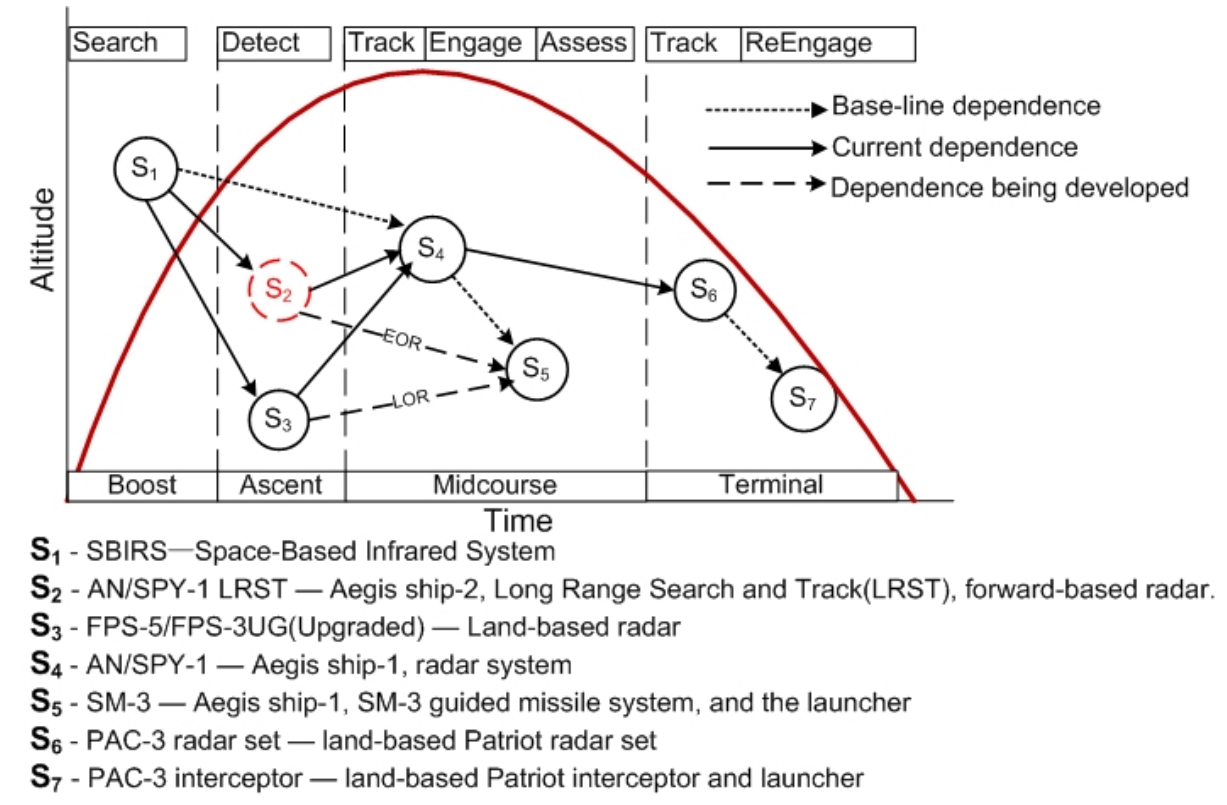

Fig. (2). Multi-layered kill chains in BMDS.

Table 1. Parameters of the functional dependency.

\begin{tabular}{|c|c|c|c|c|c|c|c|}
\hline Systems & S1 & S2 & S3 & S4 & S5 & S6 & S7 \\
\hline \hline S1 & $\backslash$ & $(0.3,80)^{\mathrm{a}}$ & $(0.4,60)$ & $(0.3,60)$ & $\backslash$ & $(0.7,0)$ & $\backslash$ \\
\hline S2 & $\backslash$ & $\backslash$ & $\backslash$ & $(0.4,60)$ & $\backslash$ & $(0.8,0)$ & $(0.9,0)$ \\
\hline S3 & $\backslash$ & $\backslash$ & $\backslash$ & $(0.3,50)$ & $\backslash$ & $(0.6,80)$ & $(0.9,0)$ \\
\hline S4 & $\backslash$ & $\backslash$ & $\backslash$ & $\backslash$ & $(0.9,0)$ \\
\hline
\end{tabular}

a. $\left(S o D_{i j}, C o D_{i j}\right)$ and “"'represents no functional dependency

the components in BMDS were represented as nodes while the links in C4ISR (Command, Control, Communications, Computers, Intelligence, Surveillance, Reconnaissance) were represented as edges. In BMDS, most component systems are able to work independently, however the function dependency further exploits the capabilities of the components. For instance, data from SBIRS relieves AN/TYP-2 of unnecessary volume or fan search, and frees the fire control radars to focus on tracking and discrimination at longer distance to remain prepared for the launch against time-sensitive targets.

For the SoS of BMDS, the probability of raid annihilation $\left(P_{R A}\right)$ is the final Measure of Effectiveness $(\mathrm{MoE})$. The capability of interceptors is defined as the probability of killing, while for sensor, it is defined as the time to detect the target.

In the combat field, it was assumed that $P_{i}$ is the probability of killing for the interceptor $P_{i}$ in an ideal situation when the operation's effectiveness is 100; however, $P_{i}$ is effected by many extra factors such as the quality of track data and the level of the training. Thus, the actual killing probability is $P_{K i}=O E_{i} * P_{i} / 100$, then $P_{R A}$ is
$P_{R A}=1-\prod_{i=1}^{n}\left(1-P_{K i}\right)$

System response time is also suggested by the authors as an $\mathrm{MoE}$ in the time-sensitive intercept task. Assuming that sensor $S_{s}$ detects the target within $T_{s}$ with 100 effectiveness, given the actual of $O E_{s}$, the response time is modified as $T_{s}=100 * T_{s} / O E_{s}$ and the response time of the first shoot is as follows:

$T_{r}=\min T_{s}$

\section{CASE STUDY}

To test the applicability of the proposed method, three notional BMDS architectures were built which took the US and Japanese BMDS as a reference [14]. Although these data were notional, the general principles and trends reflect the reality and the notional BMDS is shown in Fig. (1). Arct A is the base-line architecture based on an Aegis destroyer and a PAC-3 battery that work independently without support from other systems. Arct B is the current architecture, while Arct $\mathrm{C}$ is the architecture updated based on Arct B with AN/TPY2 radar added to introduce the functions of EOR and LOR. 
In Table 1, the independent operational effectiveness suggests that the system works without any support from others, and max capability for the sensors implies the least time to acquire a track with full support, which for interceptors means the highest probability of kill with high quality track. The functional dependency networks of BMDS architectures are presented in Fig. (2), and the notional values of $\mathrm{SoD}$ and $\mathrm{CoD}$ are given in Table 2.

\subsection{Effectiveness Analysis}

Firstly, the initial $O E$ of the systems and dependency parameters were fed into the FDN to compute the actual OE of each system. Fig. (3). shows the improvement in component $\mathrm{OE}$ due to the functional dependencies, especially in the detect and track systems. With the support of sensors, interceptors started to work $\left(O E_{i} \neq 0\right)$. Due to the networked sensor architecture, in $\operatorname{Arct} \mathrm{B}$, the $P_{R A}$ increased by $24.2 \%$, while $T_{r}$ was almost the same at about $224 \mathrm{~s}$, since the AN/SPY-1 was the key sensor to launch SM-3.

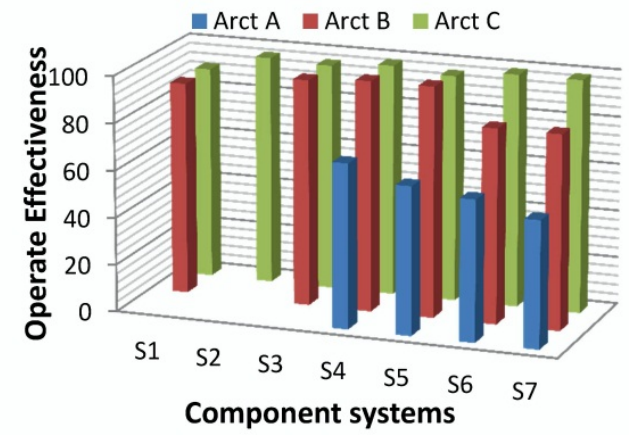

Fig. (3). Operational effectiveness of component systems.

In Arct C, the land-based AN/TPY-2 was integrated in the SOS to provide the EOR and the FPS-3 radars were updated with LOR. The BMDS made a great improvement with two intercept opportunities at $123 \mathrm{~s}$ with the probability of $61 \%$ and at 166 s with $54 \%$, as shown in Fig. (4).

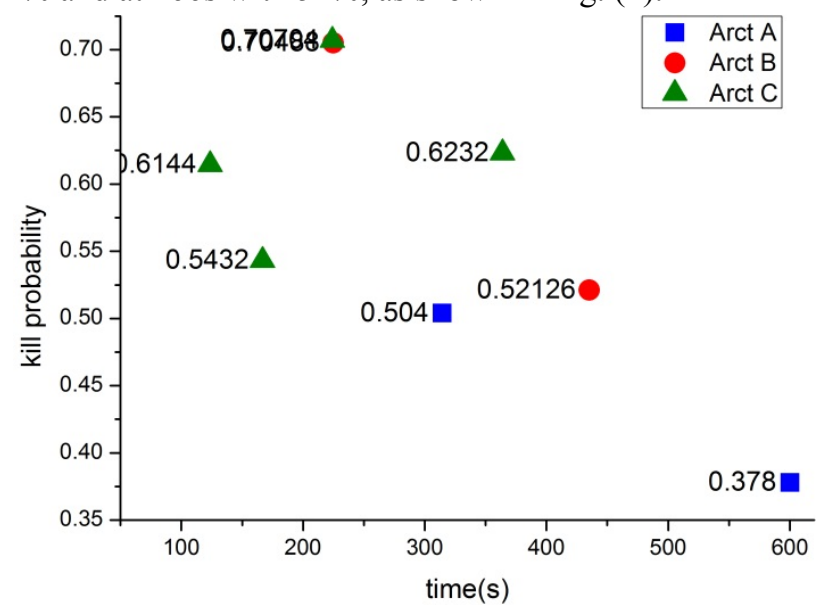

Fig. (4). Intercept opportunities.

\subsection{System Degradation Analysis}

In the battle field, the adversary would destroy or disable the components in the BMDS, for example, the anti-satellite weapons to destroy the SBIRS. On the other hand, the operational effectiveness might decrease due to the bad environment. Therefore, it is meaningful to analyse the effectiveness of BMDS under attack and system degradation.

A serious scenario was that when the SBIRS was disabled by anti-satellite weapons, other scenarios were considered for the degradation of AN/SPY-1 at three levels - $30 \%$, $50 \%,-70 \% . \quad P_{R A}$ and $T_{r}$ were compared between $\operatorname{Arct} \mathrm{B}$ and Arct C. The result is shown in Table 3, which shows that the SBIRS greatly contributed to $T_{r}$, while it made little effect on $P_{R A}$, to only about $10 \%$. The early warning was critical to the EOR engagement conducted by AN/TPY-2, as the $T_{r}$ increased by $23.9 \%(\operatorname{Arct} \mathrm{B})$ and $38.9 \%(\operatorname{Arct} \mathrm{C})$. For the

Table 2. Parameters of the component systems.

\begin{tabular}{|c|c|c|c|c|c|c|c|}
\hline Systems & S1 & S2 & S3 & S4 & S5 & S6 & S7 \\
\hline \hline Independent $O E$ & 90 & 80 & 60 & 70 & 0 & 60 & 0 \\
\hline Max Capability & $80 \mathrm{~s}$ & $160 \mathrm{~s}$ & $120 \mathrm{~s}$ & $220 \mathrm{~s}$ & 0.8 & $360 \mathrm{~s}$ & 0.7 \\
\hline
\end{tabular}

Table 3. Performance of BMDS under attack and system degradation.

\begin{tabular}{|c|c|c|c|c|}
\hline \multirow[b]{2}{*}{ Situation } & \multicolumn{4}{|c|}{ Metric for BMDS } \\
\hline & \multicolumn{2}{|c|}{ Arct B } & \multicolumn{2}{|c|}{$\operatorname{Arct} \mathrm{C}$} \\
\hline SBIRS (-100\%) & $-11.2 \%$ & $23.9 \%$ & $-9.3 \%$ & $38.9 \%$ \\
\hline AN/SPY-1 (-30\%) & $-7.9 \%$ & $18.2 \%$ & 0 & 0 \\
\hline AN/SPY-1 (-70\%) & $-25.5 \%$ & $22.3 \%$ & $-7.3 \%$ & $12.3 \%$ \\
\hline
\end{tabular}


degradation of AN/SPY-1, Arct C worked more effectively than Arct B both in the $P_{R A}$ and $T_{r}$. Arct $\mathrm{C}$ was not affected at $30 \%$ degradation of AN/SPY-1, because the land based AN/TPY-2 shared the workload of AN/SPY-1.

\section{CONCLUSION AND FUTURE WORKS}

The study proposed a new approach based on the functional dependency network to analyse the effectiveness of BMDS in view of the system of systems. Our approach overcame the limits of traditional probability models and carried out simulations by the analysis of the functional dependences between the components which built up the multilayered kill chains. The notional case study demonstrated that it was a useful approach to assess the BMDS under different scenarios.

The future works would focus on the methods to form the parameters of functional dependency which represent the effectiveness of the C4ISR, and an explorer analysis is needed to be carried out to gain comprehensive knowledge of the BMDS in different situations.

\section{CONFLICT OF INTEREST}

The authors confirm that this article content has no conflict of interest.

\section{ACKNOWLEDGEMENTS}

The research was supported in part by the National Natural Science Foundation of China through Grant Number (61273198, 91024015).

\section{REFERENCES}

[1] J. Menq, P. Tuan, and T. Liu, "Discrete Markov ballistic missile defense system modeling," European Journal of Operational Research, vol. 178, no. 2. pp. 560-578, 2007.
[2] D.A. Wilkening, "A simple model for calculating ballistic missile defense effectiveness," Science \& Global Security, vol. 8, no. 2, pp. 183-215, 2000.

[3] B.U. Nguyen, "Assessment of a ballistic missile defense system," Defense \& Security Analysis, vol. 30, no. 1, pp. 4-16, 2014.

[4] National Research Council. Making Sense of Ballistic Missile Defense: An Assessment of Concepts and Systems for U.S. BoostPhase Missile Defense in Comparison to Other Alternatives. Washington, DC: The National Academies Press 2012.

[5] R.K. Garrett, A. Steve, N. T. Baron, and J. D. Moreland Jr., "Managing the interstitials, a system of systems framework suited for the ballistic missile defense system," Systems Engineering, vol. 14, no. 1, pp. 87-109, 2011.

[6] S. Sommerer, M.D. Guevera, M.A. Landis, J.M. Rizzuto, J.M. Sheppard, and C.J. Grant, "Systems-of-systems engineering in air and missile defense," Johns Hopkins APL Technical Digest, vol. 31, no. 1, pp. 5-20, 2012.

[7] T. Ender, R.F. Leurck, B. Weaver, and P. Miceli, "Systems-ofsystems analysis of ballistic missile defense architecture effectiveness through surrogate modeling and simulation," Systems Journal, IEEE vol. 4, no. 2, pp. 156-166, 2010.

[8] O.T. Holland, and S.E. Wallace, "Using agents to model the kill chain of the ballistic missile defense system," Naval Engineers Journal, vol. 123, no. 3, pp. 141-151, 2011.

[9] P. Garvey, and A. Pinto, "Introduction to functional dependency network analysis. in the MITRE corporation and old dominion," $2^{\text {nd }}$ International Symposium on Engineering Systems, Massachusetts Institute of Technology, Cambridge, Massachusetts, 2009.

[10] C. Guariniello, and D.A. DeLaurentis, "Maintenance and recycling in space: functional dependency analysis of on-orbit servicing satellites team for modular spacecraft", In: Conference, The American Institute of Aeronautics and Astronautics, 2013.

[11] B. Drabble, "Information propagation through a dependency network model," Collaboration Technologies and Systems (CTS), International Conference on, IEEE, 2012.

[12] Y. Wang, X. Z. Wang and Q. Li, "Functional dependency network analysis of security of navigation satellite system," Applied Mechanics and Materials, vol. 522, pp. 1192-1196, 2014.

[13] W. X. Zhang, Y. Wang, and Q. Li. "An improved functional dependency network model for SoS operability analysis," Applied Mechanics and Materials, vol. 602, 2014.

[14] Japan Ministry of Defense, “Japan' s BMD”, Japan, 2009.

(C) Jian et al.; Licensee Bentham Open.

This is an open access article licensed under the terms of the Creative Commons Attribution Non-Commercial License (http://creativecommons.org/licenses/by-nc/4.0/) which permits unrestricted, non-commercial use, distribution and reproduction in any medium, provided the work is properly cited. 